\title{
Perfil epidemiológico dos pacientes atendidos no setor de fisioterapia de uma clínica- escola do interior do Ceará
}

\author{
Epidemiological profile of patients treated in the physiotherapy sector of a teaching clinic in the \\ interior of Ceará
}

Perfil epidemiológico de pacientes atendidos en el sector de fisioterapia de una clínica docente del interior de Ceará

\author{
Natália Rodrigues Macário \\ ORCID: https://orcid.org/0000-0001-5383-4090 \\ Faculdade do Vale do Jaguaribe, Brasil \\ E-mail: nataliamacario44@gmail.com \\ Jaislane Lopes Silva \\ ORCID: https://orcid.org/0000-0002-3485-4667 \\ Faculdade do Vale do Jaguaribe, Brasil \\ E-mail: jaislanelopes@gmail.com \\ Mateus Diógenes da Silva \\ ORCID: https://orcid.org/0000-0001-6334-1708 \\ Faculdade do Vale do Jaguaribe, Brasil \\ E-mail: matheusdiorge@gmail.com \\ Lilian Tayná da Silva Raulino \\ ORCID: https://orcid.org/0000-0002-6176-9054 \\ Faculdade do Vale do Jaguaribe, Brasil \\ E-mail: liliantayna13@gmail.com \\ Jorge Luiz da Silva \\ ORCID: https://orcid.org/0000-0002-1903-5292 \\ Faculdade do Vale do Jaguaribe, Brasil \\ E-mail: jorge3luiz3@gmail.com \\ Liz Soares Britto \\ ORCID: https://orcid.org/0000-0002-2810-0797 \\ Universidade do Estado do Rio Grande do Norte, Brasil \\ E-mail: lizbritto10@gmail.com \\ Daniela Maria Silva Maia \\ ORCID: https://orcid.org/0000-0002-1724-2124 \\ União Brasileira de Faculdades, Brasil \\ E-mail: danimsmaia@gmail.com \\ José Ossian Almeida Souza Filho \\ ORCID: https://orcid.org/0000-0003-1007-5172 \\ Faculdade do Vale do Jaguaribe, Brasil \\ E-mail: ossian@fvj.br \\ Roque Ribeiro da Silva Júnior \\ ORCID: https://orcid.org/0000-0001-9517-6823 \\ Universidade do Estado do Rio Grande do Norte, Brasil \\ E-mail: roquejunior@alu.uern.br
}

\begin{abstract}
Resumo
Objetivo: o presente estudo visa traçar o perfil epidemiológico dos pacientes atendidos no setor de fisioterapia de uma clínica-escola do interior do Ceará. Métodos: trata-se de um estudo transversal, quantitativo e retrospectivo. Para isso, foram analisados todos os prontuários dos pacientes atendidos no setor de fisioterapia da clínica-escola durante o ano de 2019. As variáveis analisadas foram: sexo, faixa etária, raça, profissão, diagnóstico clínico, queixa principal, segmentos acometidos, diagnóstico cinesiológico funcional (CIF) e tratamento fisioterapêutico. Resultados: observouse prevalência de indivíduos do sexo feminino (62,80\%), com idade igual ou superior aos 60 anos (31\%), de raça parda $(43,40 \%)$ e como profissão dor lar (15\%). O diagnóstico clínico mais presente foi a artrose $(8 \%)$, com queixa principal de dor $(85,80 \%)$ e o segmento mais acometido foi a coluna lombar $(32,70 \%)$. 94,70\% receberam atendimento fisioterapêutico e 83,30\% apresentavam CIF. Conclusão: o conhecimento do perfil epidemiológico permite que o fisioterapeuta reconheça as principais problemáticas que acometem a população, o tornando mais preparado para atuar tanto na prevenção como no tratamento, proporcionando, assim, melhor qualidade de vida para esses usuários.
\end{abstract}

Palavras-chave: Fisioterapia; Doenças musculoesqueléticas; Perfil epidemiológico; Clínica-escola. 


\begin{abstract}
Objective: This study aims to outline the epidemiological profile of patients seen in the physiotherapy sector of a teaching clinic in the interior of Ceará. Methods: this is a cross-sectional, quantitative, and retrospective study. For this, all medical records of patients treated in the physiotherapy sector of the school-clinic during 2019 were analyzed. The variables analyzed were gender, age group, race, profession, clinical diagnosis, main complaint, affected segments, kinesiological diagnosis functional (CIF) and physiotherapeutic treatment. Results: there was a prevalence of female individuals $(62.80 \%)$, aged 60 years or older $(31 \%)$, of mixed race $(43.40 \%)$ and working as a homemaker (15\%). The most common clinical diagnosis was arthrosis (8\%), with main complaint of pain $(85.80 \%)$ and the most affected segment was the lumbar spine (32.70\%). 94.70\% received physical therapy care and $83.30 \%$ had ICF. Conclusion: knowledge of the epidemiological profile allows physical therapists to recognize the main problems that affect the population, making them more prepared to act both in prevention and treatment, thus providing a better quality of life for these users.
\end{abstract}

Keywords: Physiotherapy; Musculoskeletal diseases; Epidemiological profile; Clinic-school.

\title{
Resumen
}

Objetivo: Este estudio tiene como objetivo perfilar el perfil epidemiológico de los pacientes atendidos en el sector de fisioterapia de una clínica docente del interior de Ceará. Métodos: se trata de un estudio transversal, cuantitativo y retrospectivo. Para ello, se analizaron todas las historias clínicas de los pacientes atendidos en el sector de fisioterapia de la escuela-clínica durante el año 2019. Las variables analizadas fueron: sexo, grupo de edad, raza, profesión, diagnóstico clínico, dolencia principal, segmentos afectados, diagnóstico kinesiológico funcional (CIF) y tratamiento de fisioterapia. Resultados: hubo una prevalencia de individuos del sexo femenino $(62,80 \%)$, de 60 años o más (31\%), mestizos $(43,4 \%)$ y que trabajaban como ama de casa $(15 \%)$. El diagnóstico clínico más común fue artrosis $(8 \%)$, con principal queja de dolor $(85,80 \%)$ y el segmento más afectado fue la columna lumbar $(32,70 \%)$. El 94,70\% recibió atención de fisioterapia y el 83,30\% tenía ICF. Conclusión: el conocimiento del perfil epidemiológico permite a los fisioterapeutas reconocer los principales problemas que afectan a la población, haciéndolos más preparados para actuar tanto en prevención como en tratamiento, brindando así una mejor calidad de vida a estos usuarios.

Palabras clave: Fisioterapia; Enfermedades musculoesqueléticas; Perfil epidemiológico; Clínica-escuela.

\section{Introdução}

De acordo com Bispo Júnior (2010), para compreender qual o papel da fisioterapia na sociedade brasileira, entender as suas responsabilidades e os seus desafios, é necessário conhecer o perfil epidemiológico da população. Diante disso, para Orsini et al. (2019), a fisioterapia é a ciência que estuda, previne, trata e reabilita distúrbios cinéticos e funcionais que podem afetar o corpo humano, sejam eles provenientes de alterações genéticas, traumáticas ou adquiridas.

Além disso, o fisioterapeuta dispõe de diversos campos de atuação, possuindo uma formação generalista. Isso permite que ele utilize conhecimentos e recursos próprios, tais como exercícios terapêuticos e a eletrotermofototerapia, como componente no tratamento de condições físicas, diminuindo o impacto de tais agravos e consequentemente melhorando a qualidade de vida desses indivíduos (Batista \& Vasconcelos, 2011).

Pensando nisso, Moreno et al. (2019) apontam que a fisioterapia dispõe de várias possibilidades de assistência e a que vem se intensificando no decorrer dos anos são os atendimentos realizados em clínicas-escolas por acadêmicos do curso de fisioterapia. Neste sentido, como instituição de ensino superior, a Faculdade do Vale do Jaguaribe (FVJ) se compromete com o desenvolvimento regional e com a melhoria da qualidade de vida no local onde está inserida. Foi fundada em 3 de julho de 1994 e oferta diversos cursos de graduação e pós-graduação, cumprindo seu papel de formação de profissionais qualificados para as exigências do mercado de trabalho. Em 12 de abril de 2018, garantindo sua excelência no ensino e compromisso social, inaugurou sua clínica-escola (Faculdade do Vale do Jaguaribe, 2020).

Nesse contexto, a clínica-escola da FVJ presta atendimentos à população em diversas áreas, servindo também como um importante campo de pesquisa. Os atendimentos, por seu turno, são realizados pelos graduandos, mediante os programas de estágio curricular dos cursos (Faculdade do Vale do Jaguaribe, 2020).

Fam e Ferreira Neto (2019), defendem que as clínicas-escolas desempenham um papel de alta relevância, não só para a comunidade, por exercer um importante papel social ao disponibilizar atendimentos gratuitos à população em geral, mas também na formação acadêmica dos estudantes. Da mesma forma, Corrêa, Arakawa e Maximino (2016), salientam que esses 
espaços formativos são ideais para o treinamento prático de habilidades adquiridas no decorrer da graduação. Além disso, a vivência nestes ambientes possibilita o contato real com os pacientes, sempre com a supervisão de preceptores. Neste sentido, faz-se necessário desenvolver a capacidade de agregar teoria e prática. Deste modo, Saldanha et al. (2014) argumentam que, enquanto ocorrem os atendimentos acontece também uma qualificação na formação acadêmica desses estagiários.

Nesse cenário, Rouquayrol (2013), aponta que a epidemiologia é definida como a ciência que estuda o processo saúde-doença, analisando a etiologia das doenças e os danos causados ao ser humano para que, a partir disso, possa elaborar planos de intervenção para o controle e erradicação de enfermidades e patologias. Além do mais, o estudo das epidemias fornece dados que podem servir como parâmetro para o planejamento de ações futuras em saúde.

Logo, esta pesquisa justifica-se pela necessidade de conhecer o perfil epidemiológico desses pacientes, uma vez que, por meio dessas informações, torna-se possível compreender e identificar as características da população. Com esse entendimento, viabiliza-se planejar assistências mais adequadas e específicas para as problemáticas apresentadas, atendendo às necessidades dos pacientes e respeitando suas particularidades, garantindo assim um melhor atendimento (Funck \& Estivalet, 2015). Além disso, o estudo pode servir como comparativo para outras pesquisas que venham a ser desenvolvidas posteriormente.

Portanto, o presente trabalho teve como objetivo traçar o perfil epidemiológico dos pacientes atendidos no setor de fisioterapia de uma clínica-escola do interior do Ceará.

\section{Metodologia}

Trata-se de um estudo transversal, por meio de investigação de pesquisa de campo, de natureza exploratória e abordagem quantitativa, com um tipo de amostra não probabilística. Segundo Fell, Pinto \& Rodriguez (2013) os estudos transversais são aqueles que capazes de investigar determinados dados ou fenômenos em um restrito período, ou seja, são mensurados em um único momento. Em relação à análise de dados, a abordagem quantitativa envolve a compreensão de dados numéricos, com base em cálculos estatísticos, o que possibilita investigar a relação entre as variáveis estudadas.

Para a base legal dessa pesquisa, foram seguidas as normas previstas na Resolução 466/12 e 510/16 do Conselho Nacional de Saúde. Assim, foram preservados os princípios bioéticos fundamentais do indivíduo (autonomia), da beneficência (incluindo a não maleficência) e da justiça. Logo, o presente estudo obteve aprovação do Comitê de Ética em Pesquisa (CEP) da Faculdade do Vale do Jaguaribe, sob o parecer $\mathrm{n}^{\circ} 4.562 .887$.

A princípio, foi realizado o levantamento de dados, que ocorreu entre os dias 01 e 15 do mês de março de 2021, por meio da seleção de prontuários dos pacientes que receberam atendimento fisioterapêutico na clínica-escola da Faculdade do Vale do Jaguaribe (FVJ), durante o ano de 2019.

Neste viés, foram incluídos 190 prontuários dos pacientes. Todavia, desses, 77 foram excluídos, já que 57 encontravam-se duplicados, ou seja, um mesmo paciente possuía mais de um registro. Além disso, 7 não apresentavam queixa principal e 13 estavam duplicados e sem queixa principal. Portanto, apenas 113 prontuários restantes foram utilizados, conforme pode-se observar no fluxograma abaixo (Figura 1). 
Figura 1 - Fluxograma.

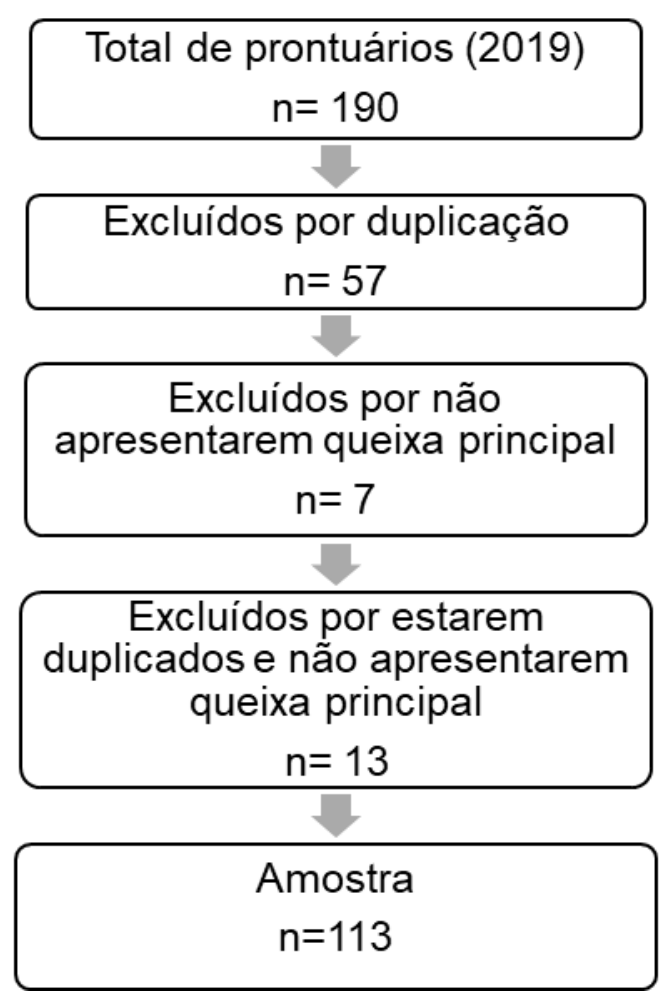

Fonte: Dados da pesquisa (2021).

Neste sentido, a coleta de dados foi realizada por meio de um formulário. Isso foi elaborado com base nas informações contidas nas fichas de avaliação fisioterapêutica presentes na clínica-escola da FVJ. Foram selecionadas para o estudo as seguintes variáveis: sexo, faixa etária, raça, profissão, diagnóstico clínico, queixa principal, segmentos acometidos, diagnóstico cinesiológico funcional (CIF) e tratamento fisioterapêutico.

Vale ressaltar que durante a coleta a identificação dos pacientes foi substituída por códigos numéricos, com objetivo de manter o anonimato dos sujeitos.

Por fim, os cômputos obtidos foram organizados em uma planilha do programa Microsoft Excel (2013), analisados mediante estatística descritiva, e apresentados em forma de tabelas para melhor explanação dos resultados.

\section{Resultados e Discussão}

A amostra foi composta por 113 pacientes de ambos os sexos, sendo que $71(62,80 \%)$ eram mulheres e $42(37,20 \%)$ eram homens. Em relação à idade, observou-se uma maior frequência de atendimentos a indivíduos com idade igual ou superior a 60 anos, representando $31 \%$ da amostra total. Em sequência, os percentuais apontam para a faixa etária de 31 a 45 $\operatorname{anos}(26,50 \%), 15$ a 30 anos $(22,10 \%)$ e 46 a 59 anos $(20,40 \%)$.

Com relação à raça, 49 dos pacientes (43,40\%) não registraram nenhuma resposta; 37 (32,70\%) se identificaram com a cor parda; 24 (21,20\%), branca; e $3(2,70 \%)$ se declararam negros.

Quanto à variável profissão, foi constatado que houve prevalência na categoria outras $(33,90 \%)$ na qual é composta em grande parte por profissionais autônomos, de serviços de limpeza e motoristas. Ademais, 25 (22,10\%) são trabalhadores domésticos, seguidos das categorias: sem registro (15\%); aposentado(a) (9,70\%); cabeleireiro(a) $(4,40 \%)$; estudante $(4,40 \%)$; artesã $(3,50 \%)$; costureira $(3,50 \%)$; pedreiro $(3,50 \%)$.

Na distribuição do perfil clínico, conforme os registros dos atendimentos dos participantes do estudo (Tabela 01), estão expostas as variáveis: "diagnóstico clínico", "queixa principal" e "segmentos acometidos". Não obstante, é importante 
ressaltar que um mesmo paciente poderia relatar mais de uma opção em cada variável.

A variável "diagnóstico clínico", conforme a área da fisioterapia onde os pacientes receberam tratamento, foi subdividida em: "traumatologia, ortopedia e reumatologia", "neurofuncional", "outros diagnósticos" e "sem registro". Na área de "traumatologia, ortopedia e reumatologia" foi observado um percentual de atendimentos de 45,40\%, abrangendo as seguintes patologias: artrose $(8 \%)$; hérnia de disco $(7,10 \%)$; fratura $(7,10 \%)$; bursite $(5,30 \%)$; tendinopatia $(3,50 \%)$; amputação (2,70\%); condromalácia patelar $(1,80 \%)$; discopatia $(1,80 \%)$; epicondilite lateral $(1,80 \%)$; luxação de ombro $(1,80 \%)$; artrite $(0,90 \%)$; fascite plantar $(0,90 \%)$; esporão de calcâneo $(0,90 \%)$; osteofitose $(0,90 \%)$; e tendinite $(0,90 \%)$.

$\mathrm{Na}$ área de "neurofuncional", observou-se um valor de 12,50\% de atendimentos das seguintes patologias: acidente vascular encefálico (AVE) (6,20\%); paralisia facial (1,80\%); doença de Parkinson (1,80\%); hidrocefalia (0,90\%); plexo braquial $(0,90 \%)$; e pé caído $(0,90 \%)$.

Na categoria "outros diagnósticos", observou-se a proporção de 2,70\% dos atendimentos divididos em: diástase abdominal $(0,90 \%)$; mastectomia $(0,90 \%)$; e hanseníase $(0,90 \%)$. E, por fim, a categoria "sem registro" abrange os prontuários que não apresentaram diagnóstico clínico, representando 53,10\% dos registros.

Com relação à "queixa principal", a mais frequente foi dor, com $85,80 \%$ do percentual, seguido de dificuldade na deambulação (7,10\%); diminuição da amplitude de movimento (ADM) (6,20\%); parestesias $(4,40 \%)$; diminuição de força (2,70\%); dificuldade nas atividades da vida diária (AVD’s) $(2,70 \%)$; fadiga (2,70\%); fraqueza muscular $(0,90 \%)$; instabilidade articular $(0,90 \%)$; e edema $(0,90 \%)$.

No que diz respeito aos "segmentos acometidos", a coluna lombar foi a mais afetada, com $32,70 \%$ das queixas. Em seguida, as reclamações de dores no ombro tiveram a expressividade de 28,30\%. Em sequência, joelho (17,70\%); coluna cervical $(9,70 \%)$; perna $(8,90 \%)$; coluna torácica $(8 \%)$; mão/dedos $(7,10 \%)$; não se aplica $(7,10 \%)$; tornozelo $(6,20 \%)$; pé $(5,30 \%)$; cotovelo $(4,40 \%)$; braço $(3,50 \%)$; punho $(3,50 \%)$; quadril $(3,50 \%)$; cabeça/face $(2,70 \%)$; e sacroilíaca $(1,80 \%)$ (Tabela 1). 
Tabela 1 - Perfil clínico dos atendimentos dos participantes do estudo.

\begin{tabular}{|c|c|c|c|}
\hline \multirow[t]{8}{*}{ Diagnóstico clínico } & \multicolumn{3}{|c|}{ Segundo a área de traumatologia, ortopedia e reumatologia } \\
\hline & & $\mathrm{n}=51$ & $45,40 \%$ \\
\hline & \multicolumn{3}{|c|}{ Segundo a área de neurofuncional } \\
\hline & & $\mathrm{n}=14$ & $12,50 \%$ \\
\hline & \multicolumn{3}{|l|}{ Outros diagnósticos } \\
\hline & & $\mathrm{n}=3$ & $2,70 \%$ \\
\hline & \multicolumn{3}{|l|}{ Sem registro } \\
\hline & & $\mathrm{n}=60$ & $53,10 \%$ \\
\hline \multirow[t]{10}{*}{ Queixa principal } & Dor & $\mathrm{n}=97$ & $85,80 \%$ \\
\hline & Diminuição de $\mathrm{ADM}$ & $\mathrm{n}=7$ & $6,20 \%$ \\
\hline & Diminuição de força & $\mathrm{n}=3$ & $2,70 \%$ \\
\hline & Fraqueza muscular & $\mathrm{n}=1$ & $0,90 \%$ \\
\hline & Parestesias & $\mathrm{n}=5$ & $4,40 \%$ \\
\hline & Dificuldade nas AVD's & $\mathrm{n}=3$ & $2,70 \%$ \\
\hline & Dificuldade na deambulação & $\mathrm{n}=8$ & $7,10 \%$ \\
\hline & Instabilidade articular & $\mathrm{n}=1$ & $0,90 \%$ \\
\hline & Fadiga & $\mathrm{n}=3$ & $2,70 \%$ \\
\hline & Edema & $\mathrm{n}=1$ & $0,90 \%$ \\
\hline \multirow[t]{16}{*}{ Segmentos acometidos } & Cabeça/face & $\mathrm{n}=3$ & $2,70 \%$ \\
\hline & Ombro & $\mathrm{n}=32$ & $28,30 \%$ \\
\hline & Braço & $\mathrm{n}=4$ & $3,50 \%$ \\
\hline & Cotovelo & $\mathrm{n}=5$ & $4,40 \%$ \\
\hline & Punho & $\mathrm{n}=4$ & $3,50 \%$ \\
\hline & Mão/dedos & $\mathrm{n}=8$ & $7,10 \%$ \\
\hline & Coluna cervical & $\mathrm{n}=11$ & $9,70 \%$ \\
\hline & Coluna torácica & $\mathrm{n}=9$ & $8 \%$ \\
\hline & Coluna lombar & $\mathrm{n}=37$ & $32,70 \%$ \\
\hline & Sacroilíaca & $\mathrm{n}=2$ & $1,80 \%$ \\
\hline & Quadril & $\mathrm{n}=4$ & $3,50 \%$ \\
\hline & Perna & $\mathrm{n}=10$ & $8,90 \%$ \\
\hline & Joelho & $\mathrm{n}=20$ & $17,70 \%$ \\
\hline & Tornozelo & $\mathrm{n}=7$ & $6,20 \%$ \\
\hline & Pé & $\mathrm{n}=6$ & $5,30 \%$ \\
\hline & Não se aplica & $\mathrm{n}=8$ & $7,10 \%$ \\
\hline
\end{tabular}

Fonte: Dados da pesquisa (2021).

Já diante a distribuição de variáveis relativas ao "diagnóstico cinesiológico funcional" (CIF) e ao "tratamento fisioterapêutico", 94 (83,20\%) dos prontuários possuem registro de CIF e diagnóstico; 7,10\% possuem somente CIF; 5,30\% estão sem registro; e 4,40\% possuem apenas diagnóstico. Já com relação ao tratamento fisioterapêutico, este foi realizado, segundo dados de 107 prontuários (94,70\%), ao passo que 5,30\% não teve tal procedimento (Tabela 2). 
Tabela 2 - Variáveis relativas ao diagnóstico cinesiológico funcional (CIF) e tratamento fisioterapêutico.

\begin{tabular}{lllr} 
Possui diagnóstico cinesiológico funcional (CIF)? & CIF e diagnóstico & $\mathrm{n}=94$ & $83,20 \%$ \\
& Só CIF & $\mathrm{n}=8$ & $7,10 \%$ \\
& Só diagnóstico & $\mathrm{n}=5$ & $4,40 \%$ \\
& Sem registro & $\mathrm{n}=6$ & $5,30 \%$ \\
Foi realizado tratamento fisioterapêutico? & & $\mathrm{n}=107$ & $94,70 \%$ \\
& Sim & $\mathrm{n}=6 \quad 5,30 \%$ \\
\hline
\end{tabular}

Fonte: Dados da pesquisa (2021).

Através dos dados desse estudo, pode-se observar que os pacientes do sexo feminino (62,80\%) foram os que mais procuraram o serviço de fisioterapia, dado esse que corrobora com a literatura consultada. No estudo de Funck e Estivalet (2015), por exemplo, observou-se uma predominância de atendimento de pessoas do sexo feminino (61\%). Da mesma forma, com proporções mais elevadas, o estudo de Rezende et al. (2015), que buscou traçar o perfil epidemiológico dos pacientes atendidos na clínica-escola de fisioterapia do Uniaraxá, demonstrou uma prevalência de atendimentos de pacientes do sexo feminino, $79,6 \%$, contra $20,4 \%$ do sexo masculino.

Em relação à idade, na presente pesquisa, pode-se verificar uma maior frequência de atendimentos para indivíduos idosos, com idade igual ou superior aos 60 anos (31\%). Do mesmo modo, o estudo de Oliveira et al. (2018) demonstrou um predomínio de pacientes idosos, com mais de 60 anos (31\%), e que a prevalência desse público pode ocorrer devido ao processo natural de envelhecimento, já que as alterações osteomioarticulares aumentam gradualmente com a idade.

No que diz respeito à raça, o estudo apontou que 43,40\% dos prontuários não apresentavam nenhum registro, evidenciando negligência em relação ao preenchimento desse dado solicitado nos prontuários. Todavia, das informações prestadas nesta categoria, é sabido que 32,70\% dos pacientes declararam-se de raça parda, o que representa a maioria. Não obstante, um estudo realizado por Silva, Lima e Leroy (2013), apontou um resultado diferente, com uma ampla participação da raça branca $(54,23 \%)$.

Ainda na pesquisa de Silva, Lima e Leroy (2013) Quanto à profissão, foi constatada a prevalência das categorias “outras", com 33,90\% dos registros, e "do lar", com 22,10\%. Comparando esses dados, pode-se concluir que a procura pelo serviço de fisioterapia varia de acordo com o local, serviço e depende das características de cada região.

No diagnóstico clínico, grande parte dos prontuários $(53,10 \%)$ encontravam-se sem registro. Todavia, a área da fisioterapia que mais recebeu pacientes foi a de traumato-ortopedia e reumatologia, sendo a artrose o diagnóstico clínico mais frequente (8\%). Com resultado aproximado, o estudo de Oliveira e Braga (2010), realizado na clínica de ortopedia da Universidade Paulista (UNIP) também apresenta a artrose (19,92\%) como diagnóstico clínico mais presente nos pacientes. A artrose, por sua vez, segundo o Department of Veterans Affairs e o Department of Defense (2020), é uma doença degenerativa e crônica que afeta as articulações cujo acometimento é mais comum em indivíduos do sexo feminino com mais de 50 anos de idade, o que consolida os dados desta nossa pesquisa, que asseveram ser a maioria mulheres com a faixa etária acima de 60 anos.

Ademais, a queixa mais frequente foi a de dor $(85,80 \%)$, tendo como segmento mais acometido a coluna lombar $(32,70 \%)$. Nesse contexto, a dor lombar é definida como uma dor musculoesquelética que se origina entre a última costela e a região glútea, sendo uma patologia muito comum que pode atingir todas as faixas etárias (Frérot et al, 2018). Por outro lado, o estudo de Oliveira e Braga (2010), realizado na clínica de ortopedia da UNIP, aponta que grande parte dos pacientes teve o 
joelho $(27,67 \%)$ como o segmento mais acometido.

$\mathrm{Na}$ maioria dos pacientes registrados nos prontuários da clínica-escola foi realizado o tratamento fisioterapêutico $(94,70 \%)$. Da mesma forma, 83,20\% também possuíam o diagnóstico cinesiológico funcional e a classificação internacional de funcionalidade, incapacidade e saúde (CIF). Este dado representa grande importância pois, segundo Alves et al. (2019), o uso da CIF permite uma maior uniformidade das informações colhidas acerca do paciente e de suas demandas funcionais, além de possibilitar uma comunicação mais padronizada entre os profissionais a respeito das características e da condição de saúde do indivíduo.

\section{Considerações Finais}

Após a análise dos dados, pode-se traçar o perfil epidemiológico dos pacientes atendidos no setor de fisioterapia da clínica-escola como sendo do sexo feminino, com idade igual ou superior aos 60 anos. Neste mesmo sentido, é possível afirmar que a maioria se declarou da raça parda e teve como principal diagnóstico clínico a artrose.

Neste viés, a área da fisioterapia mais procurada nos serviços ofertados pela clínica-escola foi a de traumato-ortopedia e reumatologia. Consequentemente, os pacientes tiveram como queixa mais frequente a dor, tendo a coluna lombar como o segmento mais acometido.

Além disso, na maioria dos prontuários analisados há registros de tratamento fisioterapêutico nos pacientes, que também possuíam o diagnóstico cinesiológico funcional e a classificação internacional de funcionalidade, incapacidade e saúde (CIF).

Logo, tendo em vista a complexidade referente à questão dor, torna-se evidente a necessidade de os cursos de fisioterapia das instituições de ensino superior (IES) oferecerem na grade curricular uma disciplina voltada especificamente para o tratamento de algias, com objetivo de melhor capacitar os futuros fisioterapeutas.

Portanto, conhecer o perfil epidemiológico da população é de suma importância, pois fornece ao profissional supracitado o reconhecimento do seu público e das problemáticas que os acometem, tornando-o mais preparado para atuar, tanto na prevenção como no tratamento de patologias, contribuindo para diminuir a incidência destas doenças e, consequentemente, proporcionando melhor qualidade de vida para os usuários. Contudo, o objetivo do presente estudo não se esgota neste artigo e as análises epidemiológicas são ainda pouco exploradas no universo acadêmico, fazendo-se, pois, necessário mais estudos nessa temática em questão.

\section{Referências}

Alves, M. T., Cavalcanti, A., Garavello, I., Kososki, E., \& Dutra, F. C. M. S. (2019). Desempenho ocupacional e aplicação da Classificação Internacional de Funcionalidade (CIF) em um serviço de reabilitação. Rev Salud Pública. 21(3), 1-17.

Batista, A. G. L., \& Vasconcelos, L. A. P. (2011). Major pain complaints of patients looking for the Physical Therapy clinic. Rev Dor. 12 (2):125-130.

Bispo Júnior, J. P. (2010). Physiotherapy and collective health: Challenges and new professional responsibilities. Cienc e Saude Coletiva. 15(1), 1627-1636.

Corrêa, C. C., Arakawa, A. M., \& Maximino, L. P. (2016). Clínica-escola de fonoaudiologia: manejo da lista de espera. Rev CEFAC. $18(5)$, $1222-1229$.

Department of Veterans Affairs. (2017). Clinical practice guideline for the management of diabetes mellitus. Department of Veterans Affairs. 8(1) 1-176.

Faculdade do Vale do Jaguaribe (FVJ). (2020). A instituição. FVJ. https://www.fvj.br/institucional/.

Faculdade do Vale do Jaguaribe (FVJ). (2020). Clínica escola. FVJ. https://www.fvj.br/campus/nucleos-de-praticas-academicas/clinica-escola/

Fam, B. M., \& Ferreira Neto, J. L. (2019). Análise das Práticas de uma Clínica-Escola de Psicologia: Potências e Desafios Contemporâneos. Psicol Ciência e Profissão. 39 (1), 1-16.

Fell, A., Pinto, M., \& Rodríguez, A. (2013). Definición de Guías de Adopción para ERP Basado en Factores Críticos de Éxito, Metodologías de Adopción y Tamaño de la Organización. Aisti. 2 (3), 1-16. 
Research, Society and Development, v. 10, n. 13, e419101321445, 2021

(CC BY 4.0) | ISSN 2525-3409 | DOI: http://dx.doi.org/10.33448/rsd-v10i13.21445

Frérot, M., Lefebvre, A., Aho, S., Callier, P., Astruc, K., \& Aho Glélé, L. S. (2018). What is epidemiology? Changing definitions of epidemiology $1978-2017$. PloS one, 13(12), 24-36.

Funck, K. T., \& Estivalet, P. S. (2015). The epidemiological profile of patients attended by the public physiotherapy service in the city of Boa Vista do Cadeado, RS TT - O perfil epidemiológico dos pacientes atendidos pelo serviço público de fisioterapia no município de Boa Vista do Cadeado, R. Fisioter em Mov. 28 (4) 685-692.

Moreno, B. G. D, Corrente, J. E, Perroca, M. G, Pavanelli, I. L, \& Rocha Júnior, P. R. (2019). Avaliação da satisfação dos usuários de fisioterapia em atendimento ambulatorial. Fisioter e Pesqui. 26 (3):322-328.

North American Spine Society. (2020). Evidence-Based Clinical Guidelines for Multidisciplinary Spine Care: Diagnosis \& Treatment of Low Back Pain. North American Spine Society. 8 (2), 1-150.

Oliveira, A. C., \& Braga, D. L. C. (2010). Perfil epidemiológico dos pacientes atendidos na clínica de ortopedia da Universidade Paulista. J Health Sci Inst, 28 (4) 356-358.

Oliveira, J. C., Santos, R. P. M. C., Calles, A. C. N., \& Monteiro, F. T. (2018). Perfil Epidemiológico Dos Pacientes Atendidos Em Uma Clínica-Escola De Fisioterapia Na Cidade De Maceió-AL. Interfaces Científicas - Saúde e Ambient. 6(2), 85-94.

Orsini, J. M. M., Silva, J. K. M., Virmond, M.C. L, \& Araujo, C. C. (2019). Evaluation of quality of life, depression and satisfaction in patients treated at the Physiotherapy teaching clinic of the State University of Northern Paraná (Uenp). Fisioter em Mov. 32(1), 1-10.

Rezende, A. L. C, Silva, R. F. O, Oliveira, L. C. N., \& Trindade, A. P. N. T. (2015). Perfil Epidemiológico Dos Pacientes Atendidos Na Clínica Escola De Fisioterapia Do Uniaraxá No Setor De Hidroterapia. Rev Odontológica Araçatuba. 36(2), 9-13.

Rouquayrol, M. Z. Epidemiologia \& Saúde. (7a ed.), Medbook, 2013.

Saldanha, O. M. F. L., Pereira, A. L. B., Medeiros, C. R. G., Dhein, G, Koetz, L.C. E., \& Schwertner, S. F. (2014). Clínica-escola: Apoio institucional inovador às práticas de gestão e atenção na saúde como parte da integração ensino-serviço. Interface Commun Heal Educ. 18 (1), $1053-1062$.

Silva, P. H. B., Lima, K. A., \& Leroy, P. L. A. (2013). Perfil epidemiológico dos pacientes assistidos na clínica de Fisioterapia Traumato-ortopédica da Prefeitura de Hidrolândia-Goiás. Revista Movimenta, Goiânia, 6 (3) 520-529. 